\title{
Pregnancy outcomes after late second trimester amniocentesis for prenatal diagnosis
}

\author{
Shauna F. Williams ${ }^{1}$, Emily Fay ${ }^{1}$, Cristina Montalvo ${ }^{1}$, Bart Holland ${ }^{2}$, Joseph J. Apuzzio ${ }^{1}$ \\ ${ }^{1}$ Department of Obstetrics, Gynecology and Women's Health, Division of Maternal Fetal Medicine, Newark, New Jersey \\ ${ }^{2}$ Department of Preventive Medicine and Community Health, Rutgers New Jersey Medical School, Newark, New Jersey
}

\section{Email address:}

joseph.apuzzio@njms.rutgers.edu (Joseph J. A.)

\section{To cite this article:}

Shauna F. Williams, Emily Fay, Cristina Montalvo, Bart Holland, Joseph J. Apuzzio. Pregnancy Outcomes after Late Second Trimester Amniocentesis for Prenatal Diagnosis. Journal of Gynecology and Obstetrics. Vol. 2, No. 4, 2014, pp. 59-62.

doi: $10.11648 /$ j.jgo.20140204.13

\begin{abstract}
Objective: To compare the rate of preterm delivery in patients who had an amniocentesis before and after 20 weeks gestation. Study Design: This is a single-center retrospective cohort study of patients referred for specialized sonogram between January 1, 2007 and December 31, 2009. Outcomes of patients who underwent amniocentesis at less than 20 0/7 weeks were compared to those who had amniocentesis on or after $200 / 7$ weeks. Results: 1041 patients met the inclusion criteria, and $290(27.9 \%)$ underwent amniocentesis. Women were younger in the late amniocentesis group and were more likely to be African-American. Gestational age at delivery, rate of preterm birth,, and birth weight did not differ between the two groups. In a multivariate analysis, gestational age at amniocentesis was not a significant factor for gestational age at delivery. Conclusion: Amniocentesis performed after 20 weeks gestation appears to be safe. This study provides reassuring information regarding late amniocentesis.
\end{abstract}

Keywords: Amniocentesis, Preterm Delivery, Prenatal Diagnosis

\section{Introduction}

Amniocentesis remains one of the most widely used diagnostic tests for chromosomal abnormalities prior to delivery. Although noninvasive screening tests are available in the first and second trimesters, these tests only provide risk-assessment data that can assist in a patient's decision to undergo an invasive test. Genetic amniocentesis has a greater than $99 \%$ accuracy in diagnosis, ${ }^{1}$ however, one risk of this procedure is pregnancy loss. The pregnancy loss rate after amniocentesis is often quoted as $0.25-0.5 \%$, as published in the recommendations by the Centers for Disease Control and Prevention in 1995. ${ }^{2}$ The 2007 American College of Obstetricians and Gynecologists Practice Bulletin quoted a procedure-related loss rate of less than 1 in $300-500 .^{1}$ Single center studies have found rates as low as $0.13 \%$ and $0.16 \%{ }^{3,4}$ Given the conflicting results, the true procedure-related loss rate in mid-trimester amniocentesis continues to be debated. Another area of uncertainty is the loss rate associated with procedures performed at later gestational ages, specifically at greater than 20 weeks gestation. Amniocentesis for genetic diagnosis is typically performed in the first half of the second trimester between 15 and 20 weeks gestation. While many studies have investigated the risks associated with mid-trimester amniocentesis, the analyses have often focused on amniocentesis performed prior to 20 weeks. ${ }^{4,5}$ In studies that have included amniocentesis performed up to 24 weeks gestation, either the outcomes after the later procedures were not specifically analyzed, or the studies were limited by small cohorts and the possibility of confounding variables. ${ }^{3,6,7}$ Therefore, the outcomes of amniocentesis performed between 20-24 weeks gestation warrant additional research since the risk after 20 weeks gestation is not spontaneous abortion but may be preterm delivery. Our objective was to determine the rate of early delivery in patients who undergo a late second trimester amniocentesis (at or greater than 20 weeks gestation) compared to early second trimester amniocentesis (less than 20 weeks gestation) and to examine any other potential differences in the populations presenting for early versus late second trimester amniocentesis in order to establish where proper education and awareness may be implemented. 


\section{Methods}

We conducted a retrospective cohort study after Institutional Review Board approval, of all gravidas referred for prenatal diagnosis and a specialized sonogram at University Obstetric Associates in Newark, NJ, which serves a large group of underinsured and uninsured patients in a northeastern urban area, between January 1, 2007 and December 31, 2009. All patients who underwent a specialized sonogram were identified by the sonogram record log and additional patient information was collected by electronic chart review. Indications for specialized sonogram included the following: advanced maternal age, abnormal quad screen, previous sonogram with a finding that increases the risk for aneuploidy or neural tube defect, and various maternal medical conditions or teratogen exposure. Patients were excluded if they carried a diagnosis of HIV, had a multifetal gestation, underwent termination of pregnancy, had a procedure at another institution, did not undergo a second trimester sonogram, medical records were incomplete, or if there was no information regarding the delivery. Amniocentesis was offered to all women and patients chose whether or not to undergo the procedure. Amniocentesis were performed under continuous ultrasound guidance by maternal-fetal medicine physicians including maternal-fetal medicine fellows. All were performed using a 22-gauge spinal needle, with approximately one milliliter per week of gestation of amniotic fluid collected. The site of the placenta was located, and transplacental puncture was avoided when possible. The amniotic fluid samples were analyzed for fetal karyotype, alpha-fetoprotein and cytomegalovirus if echogenic bowel was detected on the previous or current ultrasound. Fetal heart rate was assessed before and after the procedure.

Information collected included maternal age at delivery, ethnicity, gravidity, parity, maternal medical history, history of or current substance use, past obstetric history, indication for sonogram, age of gestation by last menstrual period, age of gestation by sonogram, sonogram findings, placenta position, amniocentesis color, amniocentesis results, quad screen results, obstetric outcome, and neonatal outcome. Indication for sonogram included advanced maternal age ( $>34$ years of age at the time of delivery), abnormal quad screen, abnormal sonogram, seizure disorder, teratogen exposure, previous sonogram with findings that increases the risk for aneuploidy or neural tube defect, maternal medical history, diabetes or at maternal request. Abnormal sonogram findings included the following: echogenic bowel, pyelectasis, choroid plexus cyst, ventriculomegaly, nuchal fold, club foot, gastroschisis, omphalocele, polyhydramnios, oligohydramnios, and intracardiac echogenic focus. Adverse neonatal outcomes were defined as spontaneous abortion, stillbirth or fetal demise, and perinatal death, as documented by Apgar scores at five and ten minutes.

Gestational age was determined by date of last menstrual period if the ultrasound dating was within 1 week when performed in the first trimester or 2 weeks if performed in the second trimester. Patients were grouped according to gestational age at time of specialized sonogram. For the initial analysis, the "late" amniocentesis group was defined as those who had amniocentesis performed at or greater than 20 weeks 0 days gestation, and our control group, or "early" amniocentesis group, was defined as those who had amniocentesis performed at less than 20 weeks 0 days gestation. The primary outcome was defined as a preterm birth less than 37 weeks. Statistical analysis was performed using Chi-square, Fisher's exact, and Kruskal-Wallis tests, with a $\mathrm{p}$ value of $<0.05$ considered significant. Multivariable logistic regression analysis included maternal age, indication for sonogram, history of past preterm delivery, and substance abuse. Included variables were predetermined because of known associations with preterm delivery.

\section{Results}

Table 1. Characteristics of patients who underwent amniocentesis

\begin{tabular}{|c|c|c|}
\hline & $\begin{array}{l}\text { Amniocentesis } \\
<20 \text { weeks } \\
\mathrm{N}=176(60.7 \%)\end{array}$ & $\begin{array}{l}\text { Amniocentesis } \\
\geq 20 \text { weeks } \\
\mathrm{N}=114(39.3 \%)\end{array}$ \\
\hline Age (years) $\dagger$ & $32.7 \pm 7.43$ & $28.4 \pm 7.16$ \\
\hline \multicolumn{3}{|l|}{ Ethnicity: * } \\
\hline African-American & $50(28.4)$ & $55(48.2)$ \\
\hline Hispanic & $107(60.8)$ & $50(43.9)$ \\
\hline Other & $19(10.8)$ & $9(7.9)$ \\
\hline Nulliparity & $52(30)$ & $39(34)$ \\
\hline Medical history & $94(53.4)$ & $62(54.4)$ \\
\hline Substance use & $3(1.7)$ & $5(4.4)$ \\
\hline $\begin{array}{l}\text { Abnormal quadruple } \\
\text { screen } \\
\text { Indication for } \\
\text { amniocentesis: } \dagger\end{array}$ & $53(38.7)$ & $41(41.4)$ \\
\hline Advanced maternal age & $92(52.3)$ & $28(24.6)$ \\
\hline $\begin{array}{l}\text { Abnormal quadruple } \\
\text { screen }\end{array}$ & $35(19.9)$ & $25(21.9)$ \\
\hline Abnormal sonogram & $22(12.5)$ & $42(36.8)$ \\
\hline History of preterm birth & $17(9.7)$ & $10(8.8)$ \\
\hline
\end{tabular}

Data presented as $\mathrm{N}(\%)$ or mean and standard deviation $* \mathrm{P}<0.05$; $\uparrow \mathrm{P}<0.0001$

Table 2. Pregnancy outcomes

\begin{tabular}{lll}
\hline & $\begin{array}{l}\text { Amniocentesis } \\
<\mathbf{2 0} \text { weeks } \\
\mathbf{N = 1 7 6}(\mathbf{6 0 . 7} \%)\end{array}$ & $\begin{array}{l}\text { Amniocenteis } \\
\mathbf{2 0} \text { weeks } \\
\mathbf{N}=\mathbf{1 1 4}(\mathbf{3 9 . 3 \%})\end{array}$ \\
\hline $\begin{array}{l}\text { Birth weight (grams) } \\
\begin{array}{l}\text { Gestational age at } \\
\text { delivery (weeks) }\end{array}\end{array}$ & $3083 \pm 854$ & $2982 \pm 740$ \\
$\begin{array}{l}\text { Preterm delivery less } \\
\text { than 37 weeks (\%) }\end{array}$ & $34(19.3)$ & $37.7 \pm 2.83$ \\
$\begin{array}{l}\text { Stillbirth/perinatal } \\
\text { death (\%) }\end{array}$ & $6(3.4)$ & $19(16.7)$ \\
\hline
\end{tabular}

Data presented as $\mathrm{N}(\%)$ or mean and standard deviation 
1251 patients were identified by the ultrasound $\log$ and $1041(83.2 \%)$ patients met the inclusion criteria (see figure 1). $290(27.9 \%)$ patients underwent an amniocentesis: 176 (60.7\%) were performed before 20 0/7 weeks ("early amnio group") and $114(39.3 \%)$ were at or after 20 0/7 weeks ("late amnio group"). Women were younger in the late amnio group $(28.4 \pm 7.16$ vs $32.7 \pm 7.42$ years, $\mathrm{p}<0.0001)$ and were more likely to be African-American (table 1). Gestational age at delivery, rate of preterm birth prior to 37 weeks, and birth weight did not differ between the two groups (table 2). Parity, substance use, and history of preterm birth also did not differ (table 1). Indication for referral for prenatal diagnosis differed with more patients in the late amnio group referred for an abnormal quad screen. When multivariate regression analysis was performed, there was no significant association of any of the included variables with gestational age at delivery.

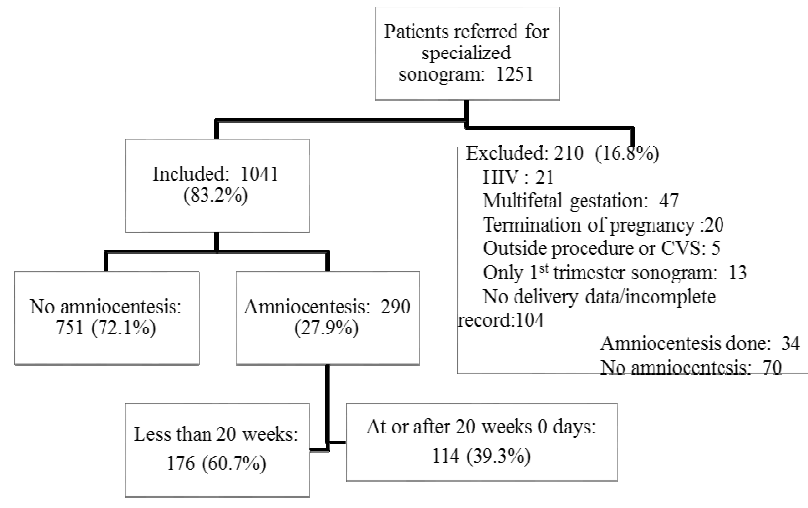

Figure 1. Study group

We repeated the analysis excluding all patients who were referred after $240 / 7$ weeks, leaving 280 patients for analysis: $104(37.1 \%)$ in the late amnio group and 176 $(62.9 \%)$ in the early group. There continued to be a disparity in maternal age and ethnicity and a similarity in gestational age at delivery and birth weight between the two groups. Multivariate analyses showed that more patients in the early amnio group delivered prior to 37 weeks as compared to the late amnio group. If we used 22 0/7 weeks as the cutoff point for defining the "late amnio" group, the significant associations were similar with no increase in preterm deliveries in the late amnio group. As with the previous analysis, the regression analysis showed an increase in preterm deliveries in the early amnio group compared to the late amnio group.

We reviewed adverse neonatal outcomes of the patients who underwent amniocentesis. The rate of adverse outcome (stillbirth, perinatal death and spontaneous abortion) did not differ between the late amnio and early amnio groups using the 20 week cut-off. There were 2 patients who experienced a spontaneous abortion and both were in the early amnio group. Three patients had pregnancies complicated by an antepartum stillbirth, two of which were associated with severe preeclampsia. Of these 3, 1 patient had amniocentesis after 20 weeks and presented at 31 weeks with severe preeclampsia and placental abruption. Two patients experienced a perinatal death, one had an amniocentesis prior to 20 weeks. The second patient was in the late group and the pregnancy was complicated by multiple anomalies.

\section{Discussion}

Genetic amniocentesis performed after 20 weeks gestation appears to be a safe procedure with preterm delivery rates, delivery gestational age and birth weights similar to when amniocentesis is performed prior to 20 weeks.

Rates of pregnancy loss and complications after amniocentesis vary in the literature. A randomized control trial from over 20 years ago reported a $1 \%$ increased risk of fetal loss with amniocentesis. ${ }^{8}$ A 2004 review of 29 studies investigating amniocentesis loss rates found an overall procedure-related loss rate of $0.6 \%{ }^{9}$ and a 2007 review showed a pooled pregnancy loss within 2 weeks of $0.6 \%{ }^{10}$ The latter review included the First and Second Trimester Evaluation of Risk for Aneuploidy (FASTER) trial in its analysis, which cited the lowest rate of fetal loss reported thus far at $0.06 \%{ }^{11}$

When looking at outcomes by gestational age, one study found that procedure-related fetal losses were significantly associated with amniocentesis performed at or beyond 18 weeks gestation compared to before 18 weeks, which the authors suggested may have been due to a difference in the background characteristics of the patients. ${ }^{6}$ Another study found higher rates of fetal loss, preterm birth, and stillbirth among women with amniocentesis performed between 19 weeks and $23+6$ weeks, compared to women who had amniocentesis performed before 19 weeks, ${ }^{7}$ but the authors acknowledge the small size of this sample and did not make any conclusion based on this.

Our study was not designed to calculate a loss rate from amniocentesis but to investigate the outcomes when amniocentesis is performed after 20 weeks gestation. We did not find a difference in preterm birth or birth weight if the amniocentesis was performed later than the traditional time period. Patients who underwent amniocentesis after 20 weeks were younger and were referred more often for abnormal quad screen results or for abnormal ultrasound findings. Considering women who have a known risk factor for aneuploidy would be referred earlier in pregnancy for specialized sonogram, these results were not suprising. In addition, we also found that African-American patients were more likely to undergo amniocentesis after 20 weeks, while Hispanic patients were more likely to have the procedure done before 20 weeks. The explanation for this is not entirely clear although later initiation of prenatal care may be a factor. The proportion of patients of advanced maternal age in this cohort does not differ by ethnicity, but there may be differences in when patients present for care and, therefore are referred later for specialized sonogram. This warrants further investigation. 
Several limitations about our results should be noted. As this is a retrospective study and we are limited to the records available, our findings may be biased due to the number of patients who were lost to follow-up. Also the sample size is limited which precludes us from making definitive conclusions. However it is unlikely that a study with a sufficient sample size for definitive conclusions will ever be done since the number of patients needed to satisfy a power analysis would be very large. We acknowledge that perhaps patients may not return for care because they experience a pregnancy loss, but we would expect this to affect both groups similarly. Additionally, because of the retrospective nature of this comparison, there may be other inherent differences not taken into account in the analysis. Given that rates of periviable and extreme preterm deliveries are rare, our negative findings may be due to the size of the cohort as indicated above.

Often patients are referred for prenatal diagnosis and amniocentesis after 20 weeks, so determining the risk of fetal loss in this time period would help tailor the discussion of the risks of the procedure with patients who may present later. Prior to 20 weeks gestation the loss is a spontaneous abortion. However after 20 weeks gestation and as the gestational age of invasive testing approaches 23 weeks or more, the loss is no longer spontaneous abortion but preterm delivery. The particular importance of this gestational age range lies in the risks of the procedure later in pregnancy which include preterm delivery and neonatal morbidities such as necrotizing enterocolitis, respiratory distress syndrome, intraventricular hemorrhage or death. Although studies investigating third trimester amniocentesis suggest a procedure-related complication rate of $0.7-1.2 \%$, including premature rupture of membranes, placental abruption, and preterm labor, among other complications, ${ }^{12-14}$ it is unclear whether late second trimester amniocentesis carries a similar complication rate. This study provides reassuring information regarding late amniocentesis and raises questions for additional research into the factors that may be associated with later initiation of prenatal care and/or referral for prenatal diagnosis.

\section{Abbreviation}

HIV: human immunodeficiency virus

\section{References}

[1] Invasive prenatal testing for aneuploidy. ACOG Practice Bulletin No. 88. Obstet Gynecol 2007; 110:1459-1467

[2] Chorionic villus sampling and amniocentesis: recommendations for prenatal counseling. MMWR Recomm Rep 1995;44(RR-9):1-12

[3] Odibo AO, Gray DL, Dicke JM, Stamilio DM, Macones GA, Crane JP. Revisiting the fetal loss rate after second-trimester genetic amniocentesis: a single center's 16-year experience. Obstet Gynecol 2008; 111:589-95

[4] Mazza V, Pati M, Bertucci E, et al. Age-specific risk of fetal loss post second trimester amniocentesis: analysis of 5043 cases. Prenat Diagn 2007; 27:180-183

[5] Towner D, Currier RJ, Lorey FW, Cunningham GC, Greve LC. . Miscarriage risk from amniocentesis performed for abnormal maternal serum screening. Am J Obstet Gynecol 2007;196:608.e1-608.e5

[6] Kong CW, Leung TN, Leung TY, et al. Risk factors for procedure-related fetal loses after mid-trimester genetic amniocentesis. Prenat Diagn 2006;26: 925-930

[7] Roper EC, Konje JC, De Chazal RC, Duckett DP, Oppenheimer CA, Taylor DJ. Genetic amniocentesis: gestation-specific pregnancy outcome and comparison of outcome following early and traditional amniocentesis. Prenat Diagn 1999;19: 803-807

[8] Tabor A, Philip J, Madsen M, Bang J, Obel EB, NorgaardPedersen B. Randomised controlled trial of genetic amniocentesis in 4606 low-risk women. Lancet 1986; 1(8493):1287-1293

[9] Seeds JW. Diagnostic mid trimester amniocentesis: how safe? Am J Obstet Gynecol 2004; 191: 607-615

[10] Mujezinovic F, Alfirevic Z. Procedure-related complications of amniocentesis and chorionic villous sampling: a systematic review. Obstet Gynecol 2007;110: 687-694

[11] Eddleman KA, Malone FD, Sullivan L, et al. Pregnancy loss rates after midtrimester amniocentesis. Obstet Gynecol 2006; 108: $1067-1072$

[12] Gordon MC, Narula K, O'Shaughnessy R, Barth WH Jr. Complications of third-trimester amniocentesis using continuous ultrasound guidance. Obstet Gynecol 2002; 99: $255-259$

[13] O’Donoghue K, Giorgi L, Pontello V, Pasquini L, Kumar S. Amniocentesis in the third trimester of pregnancy. Prenat Diagn 2007; 27: 1000-1004

[14] Stark CM, Smith RS, Lagrandeur RM,Batton DG, Lorenz RP. Need for urgent delivery after third-trimester amniocentesis. Obstet Gynecol 2000; 95: 48-50 